\title{
Natural Selection or Problem Solving. Critical Re-evaluation of Karl Popper's Evolutionism
}

\author{
Alexander Boldachev \\ Moscow, Russia \\ e-mail: alexander@boldachev.com
}

\begin{abstract}
:
Among the philosophers and the educated audience the name of Sir Karl Popper is usually associated with the critical method, evolutionary epistemology, falsification as a criterion for the demarcation of scientific knowledge, the concept of the third world and with his dislike to dialectics and contradictions. This article is aimed to show in what way all these things are connected in the evolutionary researches of the philosopher and the new conceptions, which he contributed to studying the mechanisms of evolution. Also there is an attempt to comprehend the evolutionary views of Popper, test them for falsification, relate his epistemology with his claims, which he puts forward to the theory of objective knowledge evolution and show the obvious contradiction between them.
\end{abstract}

Keywords: evolution, epistemology, selection principle, Popper.

For the dogmatic attitude is clearly related to the tendency to verify our laws and schemata by seeking to apply them and to confirm them, even to the point of neglecting refutations, whereas the critical attitude is one of readiness to change them - to test them; to refute them; to falsify them, if possible. This suggests that we may identify the critical attitude with the scientific attitude, and the dogmatic attitude with the one which we have described as pseudo-scientific (Karl Popper, Conjectures and Refutations: The Growth of Scientific Knowledge)

\section{The Tautology and Unfalsifiability of the Selection Principle}

"I have always been extremely interested in the theory of evolution and very ready to accept evolution as a fact" (Popper, 2002: 194). This is how Popper begins one of his richest in evolutionary ideas articles "Darwinism as a metaphysical research programme". In this very way according to the title of the article - he evaluated the status of Darwinism in the period of his first referring to the evolutionary topic: "I have come to the conclusion that Darwinism is not a testable scientific theory, but a metaphysical research programme - a possible framework for testable scientific theories" (Popper, 2002: 195).

And what could we expect from the author of the falsification concept? He was the one to raise a question of the scientific character of the selection principle, which is the foundation of both the traditional Darwinism and its modern interpretation - the synthetic theory of evolution. In his notes to the cited article Popper directly points out that he called Darwin's theory a metaphysical 
programme "due to the fact that it is not falsifiable" (Popper, 2002). Beside that, Popper supported another opinion which is widespread even among the evolutionary biologists - the opinion about the tautology of the selection principle:

To say that a species now living is adapted to its environment is, in fact, almost tautological. Indeed we use the terms "adaptation" and "selection" in such a way that we can say that, if a species were not adapted, it would have been eliminated by natural selection. Similarly, if a species has been eliminated it must have been ill adapted to the conditions. Adaptation or fitness is defined by modern evolutionists as survival value, and can be measured by actual success in survival: there is hardly any possibility of testing a theory as feeble as this (Popper, 2002: 199).

However, Popper did not prove his thesis about the unfalsifiliability of Darwinism and the natural selection principle either in the article mentioned above or in his other works. He only gave this explanation of the selection principle tautology. And in his late works he even made an attempt to renounce this statement (Popper, 1978) (see the analysis of this attempt below). For this reason, let us do it instead of the author of this method, by stepping to the boots of the supporter of falsification as a criterion for the demarcation of scientific knowledge.

Developing Popper's thought, we can formulate the tautology of the selection principle as follows: the statement of fact of a certain state, achieved by a system by means of this or that selection does not confirm anything except the fact that the system is not in any other state. This is the complete scientific content of the idea of "selection". In fact, the real reasons of this or that process are substituted by the selection principle. For example, to "explain" any evolutionary phenomenon within the frameworks of the theory of evolution it is considered to be enough to point out, that the natural selection was favorable to it or even simpler, that it has appeared as a result of the natural selection. If, for example, physics was built at such level of scientific rationale, then we would be content to explain the order of distribution of the liquid layers with different density in a vessel simply by some act of selection. In fact, this is absolutely true, but it has no scientific meaning without the laws of system formation, i.e. without the principles indicating not the possibility of this or that state, but the impossibility of others. A theory, which limits the realization of some phenomena, is considered to be scientific only if the predictions of this theory are testable. The theory, which states that liquid layers are distributed in a vessel in the optimum way because of the natural selection, is not scientific - its conclusions are irrefutable. This completely corresponds to Popper's idea that the laws of nature "do not assert that something exists or is the case; they deny it. They insist on the non-existence of certain things or states of affairs, proscribing or prohibiting, as it were, these things or states of affairs: they rule them out. And it is precisely because they do this that they are falsifiable" (Popper, 2005: 48).

Consequently, the selection principle does not possess the necessary property of the scientific concept - the limitation of applicability. Any scientific law is truly scientific not because it shows the possibility of existence of some phenomena (it is clear without any laws), but only on condition of proving the impossibility of the existence of the other. The energy conservation principle is valuable not because it states the feasibility of transmission and transformation of energy, but because it proves the impossibility of phenomena like perpetuum mobile. In other words, the formulation of the energy conservation principle itself supposes some options of its falsification - the phenomena, the proof of which existence will disprove this law (in our example it is the perpetuum mobile).

And has the selection principle got such options? Let us assume, that we decided to conduct a final experiment to either prove or disprove the natural selection principle. Having analyzed the environmental factors, we suppose that the A-trait will appear among the population after several generations as a result of adaptation. But after completion of the experiment we have found out that the B-trait appeared instead. Can the selection principle be disproved by that? Not at all! While 
analyzing the results, we will only conclude that the environmental factors were estimated incorrectly and the B-trait appeared in absolute correspondence with the selection principle - the adaptation took place there! So, not any result of the experiment (either A, B, C, D, or E-traits, etc.) can disprove the selection principle. The mismatch of the results and prognoses only confirms our mistakes made while analyzing the selection factors and criteria, but it does not falsify the theoretical principle itself. The attention should be drawn to the fact that the selection principle is theoretically unformalized and cannot be included into any logical constructs. Thus, the selection principle has not participated in developing the prognoses in our experiment, i.e. it has not been a part of any logical constructs. And that's why any result of the experiment can either prove or disprove it.

The same situation is with the analysis of paleontological data - any discovered empirical phenomenon, which is against the expectations, can disprove everything, but not the selection principle. The biological phenomenon itself is an evidence of its adaptiveness (or non-adaptiveness) and consequently confirms the selection principle.

We can only agree with Karl Popper's statement, that:

If we do not know how to test a theory we may be doubtful whether there is anything at all of the kind (or level) described by it; and if we positively know that it cannot be tested, then our doubts will grow; we may suspect that it is a mere myth, or a fairy-tale (Popper, 1962: 117).

And consequently, if we accept Popper's conception, the natural selection principle in its direct formulation as a statement of adaptation of the most adapted (or survival of the fittest) is not falsifiable, and that is why it can not be acknowledged as a scientific one.

\section{Selection Criteria or Problem Solving}

It is important to note, that in his very work "Darwinism as a metaphysical research programme" Karl Popper not only treated the modern evolution theory in the most critical way, but also formulated some principles and hypotheses, which could support its development. The first thing, Popper pays attention to, is the straightforwardness and unambiguity of interpretation of the selection principle in Darwinism: singling out only one criterion of its realization - the survival.

Popper himself considered the method of error elimination and revealing and solving the problems to be the main method of realization of evolutionary movement in cognition and thought, and believed that this method should be applied to every step of cognitive activity, not only to the estimation of its final result - a ready theory. And in biology the natural selection (in fact the same method as trial-and-error method) is unambiguous and linear: genome variations - selection of the fittest. In other words, obvious variability of the growth (ontogeny) of biological entities and their behavior and, consequently, the plurality of selection criteria of the ontogeny and behavioral deviations from the genetically predestined standard in traditional Darwinism are ignored. The selection criterion is always the same: survival or non-survival. If we apply this biological scheme directly to epistemology, then the natural selection in scientific cognition should be narrowed down to variations of the original hypotheses and elimination of the ready theories and not by many specific criteria, but on the general grounds of acceptance or non-acceptance. The attention should be drawn to the fact that except the elementary cases of particular adaptation of the organism (like the coloration melting into background) there is no definite selection criteria in evolutionary biology - we can only speak about the selection of the fittest. And the particular cases of adaptation (like the coloration) are "solved" by the death of the entities, which does not meet to a definite selection criterion. Seeing the mismatch between Darwin's selection principle and his own ideas of evolutionary movement mechanism, Popper supposed that: "every organism and every species is faced constantly by the threat of extinction; but this threat takes the form of concrete problems 
which it has to solve. Many of these concrete problems are not as such survival problems" (Popper, 2002: 207). Here and further in the cited article Popper primarily paid attention to the fact, that not the statement of selection itself has the scientific content, but pointing out selection criteria - the problems that are solved by selection.

Developing Popper's thought, it could be mentioned, that the natural selection is only a method of variational search for an optimal course to obtain a result, a way of solving the problem. For realization of this method it is necessary to fulfill two conditions: to have (1) a variation (a random search) of system's parameters and (2) the mechanism of testing of the obtained results against the given optimization criteria (or in Popper's terms - the definitely extracted problem).

In other words, the natural selection is first of all the selection by a criterion. Therefore, such notions as "criteria" and "the conditions of selection" should be the focal points of any theory based on the conception of selection, i.e. the "problems" itself and not the principles of their solving or methods of providing the original variability or the ways of fixing the obtained results should be the elements of the theory. The evolutionary movement should be described as a gradual interchange of the selection criteria. And it should connect these criteria into systems.

Any mention of obtaining a result with the help of selection - whether it concerns the adaptation to insignificant variations of the environmental parameters or the macroevolutional changes in the organism - first of all should be complemented with the indication of the selection criteria. In other words, if it is stated, that the system achieved a certain state with the help of some selection, the certain criteria of this selection should definitely be indicated. It is obvious, that this state is not predetermined by some random fluctuations of the elements of the system and it is not a consequence of variations, it is determined by some externally given conditions. That is to say, the realized state or some evolutionary phenomenon cannot be explained only by indicating the method or way of its achievement.

\section{The Variational Method of Problem Solving}

For further discussion of Karl Popper's evolutionary views it is necessary to make another addition concerning the nature of the selection method.

The selection is only a selection and nothing more. The selection does not have any varieties, because it is an elementary method of finding an optimal trajectory. Its content includes only a single-cut scheme: variation - the evaluation of the correspondence to the conditions of selection. And when we speak about different types of selection, we have in mind either different selection conditions and criteria (for example a propelling selection, a stabilizing selection etc.), or different objects of selection (an organism, a population, a species). In other words, the traditional phrase from the evolutionary biology texts: "many types of selection take part in the evolution of a system" in terms of standard language of scientific goal setting means that the coordinated movement of system's elements or coordination of system's separate processes is implemented through the method of variational search providing that there are several selection criteria. Such rephrasing definitely separates the method of solving from the task itself. When we state the problem like this we are to study not an "object", torn apart by the multi-directed driving forces, but the whole system, which is considered to be a variety of coordinated elements (processes). Moreover, it becomes clear that processes in the system are not one-level - they are subordinated to each other in a hierarchical way, i.e. some of them can set the selection criteria for the other ones. And the most important thing is that using this approach it is impossible to take refuge in phrases about "the supreme role of selection", its "driving creating force" etc., because it is clear, that the selection is only a method, the way the system functions and to describe its movement the selection conditions (criteria) should be indicated first of all, i.e. it is necessary to reveal the system's elements (processes) and the principles of their interaction.

In exact sciences (physics, math, etc.) the evolutionary calculations are only a computational method and do not have any theoretical meaning. Evolutionary (variational) computational methods 
are of no more importance in theoretical investigations than the other ones. Only certain results, obtained with the help of some methods, i.e. the ideas and theories, but not the methods themselves, are scientifically comprehended.

\section{The Attempt to Justify the Selection Theory}

As it was mentioned, many years after he marked the selection principle as the unscientific one, Popper tried to redeem himself:

The theory of natural selection may be so formulated that it is far from tautological. In this case it is not only testable, but it turns out to be not strictly universally true. There seem to be exceptions, as with so many biological theories; and considering the random character of the variations on which natural selection operates, the occurrence of exceptions is not surprising. Thus not all phenomena of evolution are explained by natural selection alone (Popper, 1978: 343).

However, it is noteworthy that Popper substitute the notions in this assertion: the problem of tautology and unfalsifiability of the natural selection theory is substituted by the issue of its universalism and absoluteness. It is clear, that even if it is possible to indicate some evolution phenomena, which are not connected to the natural selection in any way (by the way, Popper did not give any examples), it does not make the theory untautological and does not indicate the possibility of its falsification. The problem of the falsification of a theory should not be reduced to the indication of the phenomena, which are beyond its reach. The latter should not be confused with the phenomena, which are forbidden by this theory.

The theory, which can be falsified, should, first of all, have testable predictions: the predictions, which definitely follow from it and can be true or false, can correspond or can not correspond to the empiric data. So, the natural selection theory, as we indicated before, is not falsifiable for elementary reasons - it does not have any testable predictions. Or, to be more precise, the only testable prediction of the natural selection theory is the empiric fact of selection itself (which is tautological in essence).

However, Karl Popper suggested his version of verifiable consequence of the selection theory: "...gradualness is thus, from a logical point of view, the central prediction of the theory. (It seems to me that it is its only prediction.)" (Popper, 2002: 200). First of all, it is noteworthy, that the "graduality" cannot be considered as a consequence of the selection principle, because it is an attribute of the variational method of task solving itself. Although, we can of course present the graduality as a consequence of the theory: if the movement of a system is implemented with the help of variational approximation method, this movement is gradual. And this is a single rational conclusion, which follows from the theory - because neither the starting point, nor the direction of a system movement can follow from the method, the way of the movement implementation.

Let us analyze this single conclusion. The analysis shows, that it falsifies the selection theory - the irregularity and discretion of evolution is considered to be an admitted empiric fact. On one hand it is positive - we can conclude, that the natural selection theory is scientific (because it is falsifiable), but on the other hand we have to admit, that it is false, because its single prediction is empirically disproved. (Here it is noteworthy, that we speak about the theoretical principle, that pretend to be scientific and not about the empiric phenomenon of the natural selection, which cannot be disproved.)

Although, the problem of graduality is, of course, a problem of proportions: what seems a saltation to us, while we analyze periods of giga-years, may be a seamless transition in the time slot of life of a population. Nobody actually considers the natural selection a gradual one-dimensional process. A saltation can be described not as a speed-up of singular natural selection processes, but 
as a result of their correlation, coherence, coincidence of their directions in time, which are randomly distributed in the course of the "seamless" evolution.

However, such approach is not a justification for the natural selection theory (which was falsified while analyzing its prediction on the graduality of evolution), but a development of another theory. And the main elements of this theory should be not the selection processes themselves (which are implemented with the help of variational approximation), but a total of their criteria (goals). The analysis of the evolutionary occasions, which are only implemented with the help of selection, and, in actual fact are the conditions and criteria for the latter shall comprise the content of this theory. And this very evolutionary theory should show, why there are some concentration of evolutionary occasions, correlation of some different-level processes in certain moment of biosphere history and, as a consequence, complexly synchronized, localized in time and space evolutionary phenomena appear.

\section{Evolutionism and Natural Selection}

Before proceeding to the critical re-evaluation of the evolutionary epistemology as a whole and Karl Popper's interpretation of it in particular, I would like to make some remarks on so familiar to our ears (and minds) link of notions "evolution - natural selection".

It must be acknowledged, that a definite connection of the natural selection theory and evolution - we speak about evolution and have in mind the natural selection, and when we speak about the natural selection, we have in mind evolution - did a bad service to the evolutionism. Even creationists often do not criticize the empiric fact of gradual historical development of more and more complex biological entities (in actual fact the evolution itself), but they criticize Darwin's theory or, to be more accurate, the natural selection, its tautology and theoretical inadequacy. The creationists pursue a rather trivial strategy: they prove that natural selection and Darwinism are unscientific and then conclude that there is no evolution. And they did not actually elaborate this scheme - it was presented to them by the orthodox Darwinists, who identified evolution with natural selection.

Thanks to the orthodox evolutionary biologists the scheme "evolution - natural selection" started doing the rounds. For example, the evolutionary epistemology is called so not because it studies the evolution of scientific cognition (the evolution of cognition is a subject of any epistemology), but only because the selection principle is acknowledged in it as the mechanism and the source of this evolution. And the evolutionary cybernetics practically does not deal with any evolution - it only uses the variational and optimizing methods of calculation - i.e. the selection principle - for computer simulation of complex processes and solving the ill-formalizable tasks. It is fancy, that variational method of calculation is not called the evolutionary one in physics - in actual fact it is the same method of finding an optimal solution by varying the parameters.

Although it is noteworthy, that in evolutionary-cybernetic researches and, per se, in computer simulation of system adaptive movement, there are no phrases like "the genetic algorithm determines a certain state of programmed units population" or "the enumeration of possibilities in their behavior is the driving force and the reason of these states". The technologists (unlike the biologists) are aware of the fact that the simulated variation and selection is not more that an optimizing method of task solving and the solution is not a result of selection, but it is determined by the task itself, i.e. the initial conditions and the optimizing criteria. Although, perhaps, every time while loading the virtual population evolution program, the researchers' hope that something new and unusual will appear on the screen - something, that biologists call a result of creative activity of selection... But, alas, occurs only what must occur: the solution of an optimizing task and extraction of the most appropriate variants. And these researches undoubtedly have the scientific sense, but they correlate with evolution theory no more than aircraft dynamics correlates with navigation. 


\section{Evolutionary Epistemology or the Psychology of Creativity}

Traditional epistemology has studied knowledge or thought in a subjective sense - in the sense of the ordinary usage of the words "I know" or "I am thinking." This, I assert, has led students of epistemology into irrelevances: while intending to study scientific knowledge, they studied in fact something which is of no relevance to scientific knowledge. For scientific knowledge simply is not knowledge in the sense of the ordinary usage of the words "I know". While knowledge in the sense of "I know" belongs to what I call the "second world", the world of subjects, scientific knowledge belongs to the third world, to the world of objective theories, objective problems, and objective arguments... (Popper, 1972: 108).

It is strange to hear such arguments against traditional epistemology from Karl Popper - one of the founders of the most subjective variant of epistemology - the evolutionary one.

The focal point of the evolutionary epistemology is a trivial thesis that in science theories come and go under the influence of criticism and self-criticism. Popper said that:

The evolution of scientific knowledge is, in the main, the evolution of better and better theories. This is, again, a Darwinian process. The theories become better adapted through natural selection: they give us better and better information about reality (Popper, 1984: 239).

But he did not even mention the analysis of the evolutionary cognition as a historical progression of the intellectual innovations, the scientific ideas, which are both the driving force of formation and the selection criterion for hypotheses and theories. The evolutionary epistemology is not interested why certain scientific cognition problems appeared in a certain historic period, the main fact is, how the current scientific activity is adapted to them.

In actual fact, the evolutionary epistemology grows out of epistemology, because it focuses its attention on the method of searching for a scientific solution, on its psychological support (human curiosity, thirst for knowledge, educability, variability of thinking and behavior). Almost all problems, which are discussed in evolutionary epistemology, are the problems of psychology of creativity and scientific community sociology. And the focal point of it is not a relation of a scientific knowledge to its subject and not a rational correlation of scientific theories (different sets of knowledge), but the forms of an individual construction of knowledge and methods of its collective rating. And only the abstract method of trial-and-error without a relation to a specific content of knowledge is considered. In other words, the essence and the interconnection of evolutionary phenomena - the innovative ideas, the progression of which implements the gradual development of knowledge - are not even discussed. It is acknowledged, that to prove the evolution of scientific cognition is enough to refer to natural human curiosity, which originates from adaptive activity of animals and which makes a person to write and rewrite some texts a hundred times, and to scientific controversies, which may originate from rams butting heads on a mountain path.

However, it must be admitted, that the above-mentioned arguments are mostly of terminological character. "Evolutionism" of evolutionary epistemology depends on what we understand under the term of evolution - either it is an individual cognitive movement in a certain period of time from the germ of an idea till the completion of a theory or historical development of knowledge as a social phenomenon? However, apart from the terminological answer, there must be a solution to both problems: a careful examination of evolution of particular knowledge or theory cannot explain the nature and objective laws of scientific evolution as an integral system.

In fact, all these special aspects of the evolutionary epistemology completely correspond with their biological prototype - Darwin's theory, whose object of study in not the evolutionary phenomena (system innovations) themselves or their progression, or the objective law of their time- 
and-space distribution, but the mechanism of their local realization. And not the realization of significant evolutionary events, but small adaptations to the specific aspects of an environment. And what about the evolution of the biological system as a whole? It happens somehow gradually, maybe... If a small adaptation trait can appear as a result of a selection, then the significant changes in a system will occasionally appear someday. The most important thing that it is not forbidden by the selection theory.

The evolutionary biology deals with the biochemical support of variability rather than the historic movement of the biological system, so, in fact it is only a theory of populations' adaptation to singular environmental changes. And like this the evolutionary epistemology is interested not in the content of evolving knowledge, but in psychophysiology of its formation with the help of enumerative technique and the competitive sociology of its entities (theories) in the scientific community, i.e. the psycho-social environment, which locally provides the scientific process.

It is noteworthy, that Thomas Kuhn's shows no interest to evolution of meaningful knowledge, evolution of ideal, but it is more entitled to the name "evolutionary" than the evolutionary epistemology itself. The conception of historical alternation of "normal" and revolutionary periods of science development reflects the real evolution of scientific knowledge. Kuhn's researches we can compare with systemic evaluation of paleontological investigations in biology: to formal descriptions of biosphere history structures and the objective laws of their interchange. However, paleontology provides only empiric evidence of biological evolution, so Tomas Kuhn's epistemology indicates only the structural characteristics of the scientific knowledge history.

But we have digressed from the main topic - Karl Popper's evolutionism and his evolutionary epistemology.

\section{What is the Difference between Einstein and the Amoeba?}

The essence of Popper's evolutionary epistemology is most vividly shown while comparing Einstein's and amoeba's cognitive activity (the favorite Popper's example).

One may say, from the amoeba to Einstein there is only one step. Both work with the method of tentative trials (TT, and of error elimination EE). Where is the difference? The main difference between the amoeba and Einstein is not in the power of producing $\mathrm{TT}$, tentative theories, but in EE, in the way of error elimination. The amoeba is not aware of the process of EE. The main errors of the amoeba are eliminated by eliminating the amoeba: this is just natural selection. As opposed to the amoeba, Einstein was aware of the need for EE: he criticized and tested his theories severely (Popper, 1972: 246).

In this discourse on the methods of cognition the epistemological subject itself - i.e. the scientific knowledge - is missed. Undoubtedly, the most essential point of cognitive activity evolution (which is understood in its widest sense from the amoeba to Einstein) and, per se, of the evolution in whole is a progress in methods of achieving a result, in problem solving methods (by Popper), i.e. in the perfection of the selection principle implementation. And in the very revealing of this progress there is Karl Popper's merit. Unlike the traditional biological approach to selection, which is interpreted as a single-step act of the complete elimination of inviable deviations, Popper proved the evolution of the selection principle itself: from the simplest biological scheme of amoebas to variativity and selection in behavior of higher animals (see his version of biological evolutionary theory below) and to conscious criticism and elimination errors in a theory, but not of the entity which has made them, implemented by scientists (such as Einstein).

But in spite of the mentioned step forward in understanding the selection principle (the method of trial-and error), the example of comparison the amoeba's and Einstein's cognitive activity 
shows us, that evolutionary epistemology in its Popper's version should be related to the evolutionary methodology of knowledge. (And it is a great achievement, because in other its versions the selection methods are taken from biology without any correction or refinement). It must be acknowledged, that the epistemological essence of difference between the cognition of amoeba and scientist is not as much in a method, in conscious or unconscious selection, but in the result - in a new knowledge, in intellectual innovations. From the epistemological point of view, which, as Popper said, should be considered as "the theory of knowledge, especially of scientific knowledge" (Popper, 1972: 245) Einstein is different from the amoeba, as well as from the great majority of Homo Sapiens, because he took part in generation of the new knowledge. And here we can repeat the thought, that any epistemology should differ from psychology and scientific cognition methodology above all by its subject, should reveal the objective laws of formation of new scientific knowledge from the previous one and prove the necessity of scientific knowledge evolution and not the peculiarities of a personal or collective provision of this process.

So, let us sum up: Einstein differs from the amoeba at the level of epistemological (not biopsychological) research, because the biological entity has nothing to do with the scientific knowledge in any way, it does not produce or fix anything outside itself. The connection between a scientist and an amoeba can be seen only at the level of elementary methodology of solving the variational tasks, if there is an understanding of the essential difference between the selection criteria: in the first case the main criterion is an adequate reaction to external forces (if we speak about a separate organism) or the best possible adaptation of population to an environment, and in the second case there is a correspondence of hypotheses to a scientific idea, when we speak about an individual cognition, or the correspondence of the theory to this or that criteria of a scientific paradigm.

\section{Epistemology and Adaptation}

But, roughly speaking, almost all forms of knowledge of an organism, from the unicellular amoeba to Albert Einstein, serve the organism to adapt itself to its actual tasks, or to tasks that may turn up in the future (Popper, 1999: 64).

This thesis one more time confirms the conclusion, that Popper with his evolutionary epistemology did not solve the main task, which he set to himself even not within the scope of epistemology (the scientific cognition theory), but within cognitive methodology and psychology, within the evolutionary cognitive theory. The idea that animals (both populations and individual highly organized life forms) have cognitive ability is rather trivial. The disputable question is whether we should call the result of such activity (the result of adaptation) knowledge. The main task of any theory, which studies the cognitive evolution, is, first of all, to show the very evolution, to indicate the principle differences between the cognitive acts at different stages of evolution and not to state the permanence of mechanism and essence of cognition in the entire course of evolution. But Popper in his conclusion showed the opposite solution of such seemingly simple problem - he reduces the scientific cognition to the adaptive adjustments of cytozoon. Although, it is so clear, that Einstein's knowledge (the knowledge not of a simple man, but a genius, who developed a new theory) in no way does not "serve his organism for adaptation". Even if it is possible from any point of view to consider the scientific cognition as an adaptation, we should not do it in relation to concrete person, who produced them, and, of course, in relation to other people, but only in relation to society as a whole (and it concerns only applied, but not fundamental science).

So, Popper's evolutionary theory "links knowledge, and with it ourselves, with the cosmos; and so the problem of knowledge becomes a problem of cosmology" (Popper, 1990: 39) instead of showing the significant difference between the scientific (social) knowledge and elementary biological (genetic) one and revealing the mechanism of their transition from one to another. 
Popper duplicated the approach of the traditional biological evolutionary theory, using such methodology. The modern synthetic evolutionary theory even does not try to show the need and the regularity of principal differences between evolutionary phenomena (organisms, which have different level of complexity) and to find the differences in these phenomena implementation mechanisms or reveal the evolution of such mechanisms. Instead of this really evolutionary task, the opposite one is being solved - to show that each and every evolutionary phenomenon should be reduced to the elementary (that is not primitive, but original) biochemical reactions and the method should be narrowed down to a one-dimensional survival selection.

The arguments against Popper's epistemology, which concern the impossibility of substantiating the fact of a scientific progress or such short term of its realization (Resher, 2000: 213-214) - are the traditional arguments against any theory, based only on a selection principle (enumerative technique or trial-and-error method). But to argue about it is senseless both within the evolutionary epistemology and biological selection theory. And Karl Popper himself indicated the reason of such pointlessness - the selection principle itself as a scientific conception is tautological and unfalsifiable.

So, we could criticize Popper's evolutionary epistemology only because it is not an epistemology, not a scientific cognition theory, as he said. On one hand it does not study the substantial side of the scientific cognition, its evolution, evolution of its forms and interaction of its elements, and on the other hand all Popper's conclusions, concerning psychology and methodology of formation of knowledge relate not only to any form of human cognition (including the unscientific ones), but they extend to the cognitive activity of biological organisms, i.e. they do not concern the peculiarities of the scientific cognition. (For justice' sake it should be mentioned, that Popper himself was inclined to relate his research with the "evolutionary theory of knowledge", not with epistemology (Popper, 2000: 194), which really corresponds its subject).

\section{Karl Popper's Third World and Cognitive Evolution}

The strangest thing is, that when Popper reduces his epistemology to elementary individual and collective forms of selection, to the trial-and error method, it does not correlate with his revolutionary ideas of the third world objectiveness - an independent world, inhabitants of which "are, more especially, theoretical systems" (Popper, 1983: 439). While speaking out against the subjective epistemology, and for the objectiveness of knowledge and stating, that "epistemology should be engaged in studying the scientific problems, problem situations and scientific predictions", and that "the study of a largely autonomous third world of objective knowledge is of decisive importance for epistemology" (Popper, 1972: 111), he forgot about all these things in his evolutionary epistemology and spoke about nothing, but a role of a subject in cognitive process.

The matter is, that after conceiving Popper's conception of the third world, we should focus our attention on the objective interdependence of the scientific knowledge elements and not on the process of knowledge origin, i.e. not on the particular ways and methods of its generation. And only after it we should conduct the analysis of the way of its generation in the secondary subjective world, basing on objective and independent evolutionary laws of scientific ideas and theories. But Karl Popper seems to forgot his thesis:

An objectivist epistemology which studies the third world can help to throw an immense amount of light upon the second world of subjective consciousness, especially upon the subjective thought processes of scientists; but the converse is not true (Popper, 1972: 112),

and did "the opposite" in his evolutionary epistemology - tried to substantiate the evolution of scientific cognition, basing at its subjects' actions. 
And just by referring to the conception of the third world, we can find out the epistemological difference of the amoeba's and Einstein's cognitive activity. Popper came close to solving of the problem and stated, that the third world "has a strong feed-back effect upon us; that is to say, upon us qua inmates of the second and even of the first world" (Popper, 1972: 112), but he did not directly connect this effect with the element of his cognitive scheme - error elimination (EE). Yes, there is a difference between the error elimination by elimination of the subject itself and the critical analysis, but it is a formal difference, which relates the way of the movement implementation, not to its nature, i.e. it does not relate to epistemology as a scientific cognition theory. And it is clear, that the most important thing in error elimination is not the form of its implementation, but the criterion, according to which it is implemented. For Einstein this criterion is formed under the influence of Popper's world of objective knowledge and manifested as an idea, which precedes the selection act. And not only the amoeba, but also the majority of people, which are not engaged in science can not have the criterion of elimination of an incorrect hypothesis. The amoeba has its own world of objective "knowledge" - evaluation indicator of behavioral acts ("hypotheses").

Actually, such development of Karl Popper's ideas really give us the key to building a subjectless epistemology - all acts of individual and collective "error elimination" are presented not as basic driving force of science, but as local implementation and objectification of ideas as the selection criteria. Here we should definitely distinguish on one hand (1) the objective existence of ideas and knowledge, and on the other - (2) the knowledge generation process, implemented by way of selection of hypotheses and theories, according to the objective criteria, formulated in the third world. Or, if to express the same in Karl Popper's words:

We should constantly be aware of the distinction between problems connected with our personal contributions to the production of scientific knowledge on the one hand, and problems connected with the structure of the various products, such as scientific theories or scientific arguments, on the other (Popper, 1972: 114).

It is clear, that from such point of view on cognitive evolution, the selection procedure itself has only supportive meaning, being not more than a form of objective ideas movement implementation. Man (a scientist) fulfills a double function in this scheme of scientific cognition: on one hand he is generator of ideas and detector, which filters them basing on some transcendental idea or criterion, and on the other hand - integrator of world of objective knowledge, who extracts ideas and criteria from it in process of learning and studying a problem.

... New problems, - as Popper wrote - arise from our own creative activity; and these new problems are not in general intentionally created by us, they emerge autonomously from the new relationships which we cannot help bringing into existence with every action, however little we intend to do so (Popper, 1979: 119).

If we paraphrase these general conclusions on Popper's ideas in biological terms, we can say, that the evolutionary theory of biological phenomena can and should be presented as a theory of a gradual interchange of selection criteria, in which the organisms and populations are the cognitive, integrative and realizing subjects. And the natural selection and the forms of its implementation (genetic, ontogenetic and behavioral mechanisms) should join the theory as the variable methods of evolutionary movement implementation. "A random movement is accepted when it fits into the higher level structure, - as Popper wrote- otherwise it is rejected" (Popper, 1977: 147). 


\section{Karl Popper's Evolutionary Theory}

As it was already mentioned, Karl Popper's evolutionary approach (both in cognitive theory and in biology) suggests a lot of selection criteria, that is problems that should be solved - and, the most important is the multilevelness and hierarchicalness of these problems, and, consequently, of their solutions. Popper stated that solving the more long-term problems (of high generality) not only precede the particular solutions, but does not recede to them (Popper, 2000: 208). The traditional biological evolutionary theory is based on a strict succession of adaptations and, therefore, presents the significant systemic changes in organism as a result of succession of small adjustments and narrows all selection criteria down to survival.

Popper tried to take Darwinian conception away from an endless circle of tautology, tried to break a self-referent chain: in the course of natural selection survives the most adapted, and the most adapted is that which survives in the course of natural selection. He included into this chain a lot of selection criteria, which he called the problems solved by the trial-and error method.

In our system, not all problems are survival problems: there are many very specific problems and sub-problems... Our schema allows for the development of erroreliminating controls... that is, controls which can eliminate errors without killing the organism; and it makes it possible, ultimately, for our hypotheses to die in our stead (Popper, 1979: 244).

But, unfortunately, Karl Popper did not go further than stating the diversity and hierarchy of the selection aims (problems and their solutions). Although he made a step forward in biological evolutionary theory, by suggesting his version in his article "Darwinism as a metaphysical research programme".

Karl Popper based his "suggestions for an enrichment of Darwinism which might explain onto-genesis" (Popper, 2002: 201) on two points: (1) the statement, that the directional movement of biological evolution cannot be explained on the basis of single-level selection scheme, single selection criterion and solving of one only problem - the survival; and (2) the conclusion, that the total of selection criteria (problems) makes the hierarchy and solution of the highest (behavioral) level problems can directly influence the selection criteria at the lowest anatomical level, directing and fastening its evolution.

Popper suggested to distinguish

external or environmental selection pressure from internal selection pressure. Internal selection pressure comes from the organism itself and, I conjecture, ultimately from its preferences (or "aims") though these may of course change in response to external changes (Popper, 2002: 201).

At first glance the scheme of his evolutionary concept is rather simple: (1) changes in behavior ("preferences") of organisms (in the course of generations), caused by changes in external conditions, which are implemented without any changes in a genome; (2) can promote the selection of the organisms, which have "skills", supporting these behavioral changes, and, as a final result (3) lead to genetic fixation of anatomic traits, meeting the requirements of new "preferences" which act as new selection criteria, as new problems at an anatomic level.

Actually, Popper stated, that the cognitive behavioral activity, which is implemented without any changes in a genome, can set the selection criteria for morphology modifications and determine the direction of selection.

...Every behavioral innovation by the individual organism changes the relation between that organism and its environment: it amounts to the adoption of or even to 
the creation by the organism of a new ecological niche. Thus the organism, by its actions and preferences, partly selects the selection pressures which will act upon it and its descendants. Thus it may actively influence the course which evolution will adopt. The adoption of a new way of acting, or of a new expectation (or "theory"), is like breaking a new evolutionary path (Popper, 2002: 210).

Popper explained his "prediction about such internal selection principle" in a form of a diagram: $\mathrm{p}>\mathrm{s}>\mathrm{a}$ ("the preference structure and its variations control the selection of the skill structure and its variations; and this in turn controls the selection of the purely anatomical structure and its variations" (Popper, 2002: 203)).

But neither philosophers, nor biologists accepted this conception of a multi-level hierarchic selection. Biologists acted like that for quite objective reason: it was based on a genetically false prediction, that there are some genes, which are separately responsible for "preferences" (p), "skills"(s) and autonomy (a).

However, it is easy to bring the conception of the multi-level selection and the interdependence of its criteria (the solved problems in Popper's terms) to correspondence with the modern biological data. We should only consider a trivial thesis, that every level of selection - for example, behavioral, organismic (ontogenetic) and cellular (genetic) - has the particular mechanisms of providing the selection: the particular variable parameters and forms of fixation of the result. Behavioral variations appear in several generations with the help of training and imitation. The morphology of an organism can vary within acceptable variants of ontogeny and appear in generation through the influence of maternal organism on embryo (see the epigenetic evolutionary theory (Shishkin, 1998)) - and the ontogenetic selection goes without any changes in genome as well as on a behavioral level. And the selection based on a standard genetic mechanism occurs only at cellular level - or, more specifically, at cellular reproductive line. In fact, Popper's conception narrows down to stating the existence f several relatively independent selection levels, every higher one of which can be considered as an external environment for the lower one: ontogeny adapts to the behavioral environment and the cellular reproductive line adapts to the ontogenetic changes. It is natural, that the adaptation of every lower level goes in direction of providing the best possible support and providing the reproduction of a higher level phenomenon, which, as a final result, should gradually lead to genetic fixation of behavioral phenomena. (To learn more about the conception of level selection see (Boldachev, 2007: 138-150)).

Philosophically generalizing the expounded conception of level selection in Karl Popper's terms, it can be concluded, that the problems, created by biological organism by its behavioral activity are a far more significant and strong selection criterion for the ontogenetic ways, and, consequently, the genetic selection than an abstract environment. And to explain the trend of evolution is possible only considering a hierarchy of interrelated problems.

But let us give word to Popper himself:

Thus men like Butler and Bergson, though I suppose utterly wrong in their theories, were right in their intuition. Vital force ("cunning") does, of course, exist - but it is in its turn a product of life, of selection, rather than anything like the "essence" of life. It is indeed the preferences which lead the way. Yet the way is not Lamarckian but Darwinian (Popper, 2002: 209).

\section{References}

1. Boldachev, A.V. Novation. Judgments in Line with the Evolutionary Paradigm, (in Russian). St. Petersburg: Publ. House of St. Petersburg University Press, 2007.

2. Popper, K. Conjectures and Refutations: the Growth of Scientific Knowledge. London: Routledge and Kegan Paul, 1962. 
3. Popper, K. Epistemology Without a Knowing Subject, [in:] [6].

4. Popper, K. Natural Selection and the Emergence of Mind. First Darwin Lecture, Darwin College, Cambridge, 1977, [in:] Evolutionary Epistemology, Rationality, and the Sociology of Knowledge, eds. G. Radnitzky and W.W.W. Bartley, III. Open Court 1987.

5. Popper, K. Natural selection and the emergence of mind, Dialectica 32, 1978, 339-355

6. Popper, K. Objective Knowledge: An Evolutionary Approach, Revised Edition, Clarendon Press, 1979.

7. Popper, K. Evolutionary Epistemology, [in:] Popper K. Evolutionary Theory: Paths into the Future. London: John Wiley \& Sons, 1984.

8. Popper, K. Towards an Evolutionary Theory of Knowledge, [in:] Popper K. A World of Propensities. Bristol, Thoemmes, 1990, 27-51.

9. Popper, K. All Life is Problem Solving All Life Is Problem Solving. London: Routledge, 1999.

10. Popper, K. Unended Quest: An Intellectual Autobiography. London, New-York: Routledge, 2002.

11. Popper, K. The Logic of Scientific Discovery. London and New York: Routledge / Taylor \& Francis, 2005.

12. Rescher, N. Peirce, Popper and the Methodological Turn, [in:] Rescher Nicholas. Peirce's Philosophy of Science, ch.2, part 2. Notre Dame, London: University of Notre Dame Press, 1978.

13. Shishkin, M.A. Evolution as epigenetic process, Sovremennaya paleontologiya [Modern Paleontology] (in Russian). Moscow: Nedpa, 1988, vol. 1, pp. 142-169. 